\title{
Recuperação
}

\section{de informação}

\section{baseada em} clusters

\section{INTRODUÇÃO}

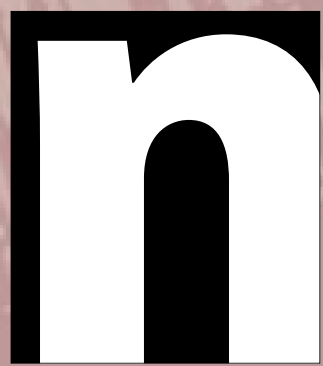

os últimos anos a internet se consolidou como a principal fonte para quem busca informação, seja informação acadêmica, de divulgação, comercial, de entretenimento ou de outra natureza. Para possibilitar e facilitar esse processo de busca, praticamente todos os portais e sites da web oferecem ferramentas, motores, mecanismos ou interfaces de busca que pretendem levar o usuário à informação desejada.

Há sites especializados em buscar,como é o caso do Google, o buscador mais conhecido da internet, e outros como o Yahoo, o Ask, o Lycos e, mais recentemente, o Amazon, com seu mecanismo de busca 
A9. Os portais buscadores surgiram logo após o aparecimento da internet com a intenção de prestar um serviço extremamente importante: a busca de qualquer informação na internet, apresentando os resultados de uma forma organizada, e também com a proposta de fazer isso de uma maneira rápida e eficiente.

$\bigcirc$ que caracteriza um site ou portal buscador é o funcionamento de seu motor de busca. Esse motor rastreia a informação disponível na web, periodicamente, navegando de página em página, ou de site em site, extraindo os documentos, as palavras, os termos que melhor representam a informação capturada, e então a armazena em uma gigantesca base de dados que pode ser consultada pelos usuários através de uma interface de busca. Portanto, os buscadores funcionam na dependência de coleções de fontes de informação não produzidas, geridas ou organizadas por eles. Sua especialidade é buscar em fontes de informação disponíveis na internet, independente de quem as provê. Assim, os buscadores se mostraram imprescindíveis para o fluxo de acesso à informação e para a conquista de novos usuários e visitantes para os sites na internet.

\section{BUSCADORES VERSUS BIBLIOTECAS VIRTUAIS}

\section{CARMEN VERÔNICA MENDES ABDALA}

é bibliotecária da Bireme/Opas/OMS.

\section{VINÍCIUS ANTÔNIO} DE ANDRADE

é analista de sistemas da Bireme/ Opas/OMS.
Praticamente todo site ou portal tem sua interface e motor de busca com a mesma função, que é facilitar o acesso à informação, mas a diferença principal está no domínio da coleção de fontes de informação buscada. Comparando os buscadores com sites de bibliotecas virtuais, como a BVS (Biblioteca Virtual em Saúde - http://www.bvsaude.org), 
de portais Opac (Online Public Access Catalog - catálogos on-line de bibliotecas), como o portal SIBi-Net da USP (Sistema Integrado de Bibliotecas da Universidade de SãoPaulo-http://www.usp.br/sibi), ou ainda de portais de periódicos científicos, como a SciELO (Scientific Electronic Library Online - http://www.scielo.org), estes últimos estão suportados, principalmente, por suas próprias coleções de fontes de informação, que são bases de dados referenciais, serviços de informação, acervos das bibliotecas, etc.

Há algum tempo não distante, parecia haver uma resistência em aceitar os portais buscadores ou, no mínimo, uma relação de distância por parte dos provedores de sites ou portais acadêmicos. Por um lado, tínhamos a limitação dos buscadores em localizar toda a informação disponível na internet, limitação dada pelo funcionamento do motor de busca, que não chegava aos conteúdos e informação disponíveis em páginas dinâmicas e, por outro lado, tínhamos os provedores de fontes de informação que não preparavam os seus conteúdos para serem recuperados pelos motores dos buscadores.

Isidro Aguillo e outros autores mencionavam que os buscadores cobriam uma parte dos conteúdos e informação disponíveis na web, deixando "invisível" uma parte considerável de informação correspondente ao que está em bases de dados, entre outros recursos e fontes de informação.

A situação deveria fortalecer as interfaces de busca dos portais acadêmicos, que oferecem acesso à informação organizada em bases de dados, catálogos coletivos e outras fontes de informação referencial, considerada informação valiosa, até então não rastreada pelos motores de busca por não ser indexada por esses motores.

Mas esse cenário mudou e vem mudando de forma acelerada. Novos desenvolvimentos foram implementados nos motores de busca; foi criado o Google Scholar, os recursos de apresentação dos resultados por relevância e a clusterização são tendências importantes, que facilitam ainda mais a busca de informação na internet; e os portais e sites provedores de informação, em geral, passa- ram a organizar suas fontes de forma a abri-las para os diferentes motores de busca.

\section{PROMOVENDO A VISIBILIDADE DA INFORMAÇÃO}

Com a percepção ampliada de que não basta que a informação esteja disponível na internet, mas que ela precisa ser identificada e buscada por diferentes interfaces e motores de busca, é fundamental garantir que os conteúdos e a informação armazenados nas coleções dos portais ou sites web sejam indexados pelo Google, por outros portais buscadores e de serviços de informação, tantos quantos forem possíveis.

Citamos o exemplo do portal SciELO Brasil, uma coleção de periódicos científicos brasileiros que, até o ano de 2005, não era buscada pelo Google, com textos completos dos artigos, embora operando em compatibilidade com o protocolo OAI (open archives initiative), o que permitia livre acesso aos metadados da coleção de provedores de serviço open archives de todo o mundo. A SciELO era parte da informação invisível para os buscadores.

A partir do ano de 2006, o Google Scholar passou a indexar os artigos do portal SciELO Brasil, graças a uma preparação dos dados. Com isso, o número de visitas ao portal SciELO Brasil, que em 2005 registrou uma média mensal em torno de 3 milhões de artigos acessados com texto completo, teve um incremento de $180 \%$. Em 2008, a SciELO Brasil registra uma média mensal de 8 milhões de acessos ao texto completo de artigos, sendo que cerca da metade chega por meio do Google Scholar.

Atualmente, um artigo SciELO pode ser recuperado por diferentes interfaces $\mathrm{e}$ motores de busca, inclusive pela interface de busca do próprio portal SciELO, como ilustrado na figura da página seguinte.

A ampliação da visibilidade da informação na internet não é possível sem que haja inovaçãoe desenvolvimento nos sistemas de informação. Nesse sentido, é importante que esses desenvolvimentos sejam orientados 
FIGURA 1

Acesso a artigos SciELO por diferentes motores ou interfaces de busca

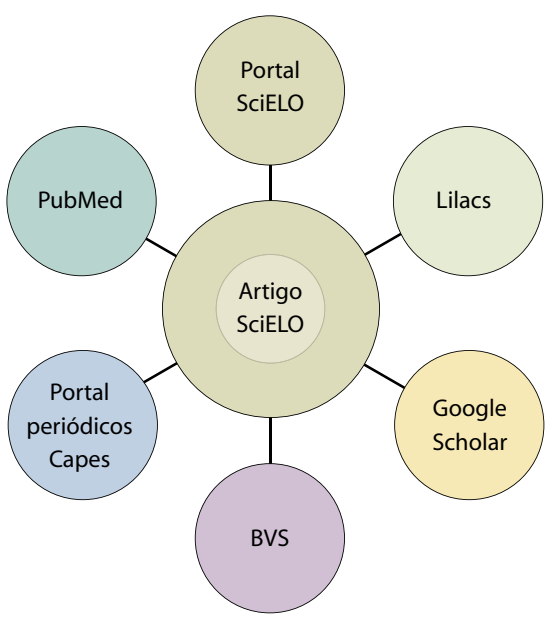

para uma arquitetura da fonte de informação e construção de redes de fontes e fluxos de informação baseadas em componentes, protocolos e processos que maximizem as possibilidades de operação na web.

O modelo metodológico da SciELO e da Biblioteca Virtual em Saúde aplica esse conceito em uma arquitetura baseada em três níveis separados de processamento:

- nível dado-contém os arquivos ou bases de dados com os registros de conteúdos. Os arquivos de dados estão acessíveis e são baseados em protocolos de acesso aberto para serem indexados por diferentes buscadores (indexadores);

- nível índice - contém os arquivos de índices para recuperação da informação (dado). Essa camada pode ser representada por diferentes indexadores aplicados aos mesmos arquivos de dados;

- nível interface e webservices - em geral opera em páginas web e acessa os índices para recuperação e navegação nos conteúdos. As interfaces são ilimitadas, tanto em quantidade quanto em formato de apresentação, e podem ser portáveis para navegadores $w e b$, telefonia móvel, TV digital, etc. Énesse nível que se disponibiliza o dado usando protocolos-padrão da internet, como RSS (really simple syndication), XML (extensible markup language) e OAI(open archives initiative), permitindo a sua indexação por outros motores de busca e interfaces.

$\mathrm{O}$ que caracteriza essa arquitetura de fonte de informação é a independência dos níveis, os quais podem operarem diferentes servidores, diferentes sistemas computacionais, com diferentes designs e concepção de interfaces. É o mesmo conteúdo, a mesma informação, sendo disponibilizadoem diferentes produtos e serviços de informação, e visível por diferentes interfaces de busca.

A aplicação dessa arquitetura para desenvolvimento de produtos e serviços, aplicações, websites e portais, permite estabelecer mecanismos de operabilidade entre diferentes aplicativos e sistemas de informação, baseados na componentização, baixo acoplamento ou independência entre os componentes dos aplicativos, portabilidade, etc. Encoraja e facilita a reutilização de serviços e produtos de informação, assim como a refatoração ou melhoria contínua no seu desenvolvimento.

Para as bibliotecas virtuais, Opacs e outros portais de informação, essa arquitetura, especialmente o nível de webservices, não somente abre as suas coleções de conteúdos e fontes de informação para outras inter-

\section{FIGURA 2}

Arquitetura de informação baseada em níveis de processamento

Rede de interfaces e webservices (RSS, OAI, XML etc.)

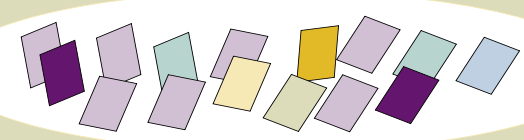

Rede de índices (Isis, Google, Collexis, etc.)

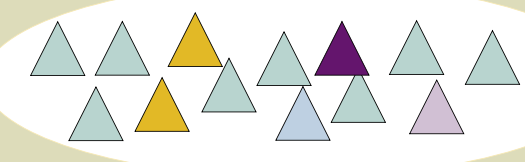

Rede de fontes de informação 
faces e motores de busca na internet, mas possibilita o acesso integrado a conteúdos e informação disponíveis por outros provedores de fontes de informação, a partir de suas próprias interfaces de busca. Concretamente, a coleção SciELO, por exemplo, pode ser recuperada por qualquer interface de busca, de forma integrada com outras coleções e acervos de bibliotecas.

\section{NOVOS RECURSOS DE BUSCA NA INTERNET}

Conectar os usuários com o conteúdo de que eles precisam, quando precisam, não é mais opcional. É uma necessidade que deve ser atendida através das interfaces e dos motores de busca que operam em sites e portais da internet. Os usuários têm à sua disposição uma variedade e quantidade imensuráveis de fontes de informação a ser buscada na internet, além de uma grande opção de interfaces com diferentes tipos de recursos e sofisticação nos processos. Esses usuários esperam, cada vez mais, resultados de alta qualidade para a sua busca, e interfaces que os ajudem a encontrar a informação que estão procurando de maneira precisa, rápida, fácil e organizada.

Ao mesmo tempo, essa realidade coloca um novo problema para os usuários em geral, que precisam aprender a usar e a distinguir entre os diferentes tipos, qualidade e fontes de informação.

Os buscadores e a maioria dos sites e portais da internet oferecem uma interface com recurso de metapesquisa ou metabusca, oque permite ao usuário submeter uma mesma expressão de busca para ser processada em várias fontes de informação (bases de dados), simultaneamente.

Entretanto, devido aos diferentes tipos de conteúdos das fontes de informação, estruturas de campos de dados e formatos variados de apresentação da informação, a maior parte das bibliotecas virtuais e Opacs ainda não apresenta o resultado dessa busca de forma integrada, em uma única lista. $E$ há aqueles que nem sequer oferecem o recurso de metabusca. O usuário precisa entrar em fonte por fonte de informação para ter acesso à informação recuperada em cada uma delas.

O Google e outros buscadores não têm esse problema, e por essa razão, entre outras, o ambiente dos usuários de bibliotecas acadêmicas tem mudado drasticamente nos últimos anos, e os motores de busca da internet têm se tornado a ferramenta preferível no lugar dos catálogos coletivos e bibliotecas virtuais para encontrar informação.

Tradicionalmente, o resultado de busca em serviços de informação especializada é mostrado em um ordenamento cronológico reverso (a partir da data mais recente), como padrão, com a possibilidade de ser alterado para nome de autor, título, revista ou outros campos de dados, dependendo do tipo da fonte de informação.

A hierarquização do resultado da busca por relevância foi introduzida nas interfaces de busca como recurso para ordenar a sequiência de apresentação dos registros (ou referências) na lista de resultado, representando uma tentativa de separar o joio do trigo. $\mathrm{O}$ algoritmo usado para fazer o ranking por relevância está concebido para determinar que documento ou documentos melhor se aproximam do assunto representado pela expressão de busca do usuário. Os diferentes motores de busca e interfaces usam diferentes algoritmos de ranking, normalmente não revelados. Assim, pode haver diferença de ranking de um sistema para outro, dentro de um mesmo conjunto de informação, o que mostra claramente que a relevância está nos olhos de cada um dos sistemas, segundo observou Jacsó, em 2005.

A metabusca com a apresentação do resultado em uma única lista, ordenada por relevância, consolidou o sucesso dos buscadores na internet.

Entretanto, como muitos autores já afirmaram, a evolução da web muda esse cenário constantemente, e a necessidade de melhor apresentação dos resultados recuperados em milhões, depois bilhões, de páginas web não estruturadas e não classificadas se tornou óbvia. Surge um novo recurso que 
contribui para a organização mais eficiente do resultado de uma busca com opções que ajudam o seu refinamento, chamado de clustering, em inglês, e de "clusterização" ou "agrupamento", em português.

A “clusterização" é a classificação de objetos em diferentes grupos ou, mais precisamente, a partição de um conjunto de dados em subgrupos (clusters). Atualmente, um dos sistemas de pesquisa com "clusterização" mais conhecidos e aplicados é o da empresa Vivisimo.

Como exemplo de aplicação de uma busca integrada, com apresentação de resultado por relevância e com "clusterização", apresentamos a experiência da Biblioteca Virtual em Saúde, que tem uma interface de busca suportada pelo sistema iAHx, também inspirado no modelo de clusterização Vivisimo (http://vivisimo.com).

\section{iAHx - SISTEMA DE}

\section{PESQUISA INTEGRADA COM CLUSTERIZAÇÃO}

O iAHx é um sistema de pesquisa integrada que foi desenvolvido pela Bireme com o objetivo de aperfeiçoar o mecanismo de apresentação do resultado de busca da Biblioteca Virtual em Saúde e de sua coleção de fontes de informação, possibilitando visualizá-la de forma integrada, individualizada e ordenada por diferentes critérios e clusters.

Alguns motivos impulsionaram odesenvolvimento do iAHx. Do lado do usuário, havia uma excessiva quantidade de passos (ou cliques) até chegar à informação demandada; uma curva de aprendizagem lenta em função das diferentes interfaces oferecidas para busca nas fontes de informação que fazem parte da coleção da BVS, além dos variados formatos de apresentação do resultado da busca, praticamente um formato para cada fonte de informação; e uma oferta de serviços diferenciados e não uniformizados. Do lado do provedor do portal, um alto custo de operação e atu- alização dos sistemas; alta dependência do serviço de capacitação de usuários para uso eficiente dos recursos de busca oferecidos; e, principalmente, não atendia ao modelo conceitual de arquitetura da informação em três níveis de processamento.

Nos dias de hoje, essa situação ainda está presente na maioria dos portais e sites acadêmicos brasileiros e dos sistemas e serviços de informação científica e técnica.

Através da Figura 3 temos uma mostra da forma de apresentação do resultado de uma busca processada com o sistema iAHx, com a "clusterização" e outros recursos.

Esse exemplo de busca ("aleitamento materno Brasil") foi processado em toda a coleção da BVS, que inclui as fontes de informaçãodestacadas noquadro aoladodireitoda figura; o resultado da busca (564 referências bibliográficas) está apresentado em ordem de relevância, ou seja, os documentos que mais se aproximam da busca são mostrados nas primeiras páginas (de 10 em 10) e não por ordem de data mais recente como no modelo anterior. Podemos notar que a primeira referência apresentada para essa busca é do ano de 2003 e a segunda do ano de 2008. O usuário tem a opção de mudar a ordem de apresentação do resultado se desejar.

Ao lado esquerdo da figura está a "clusterização" do resultado para essa busca. Os

FIGURA 3

Apresentação "clusterizada" de resultado de pesquisa - iAHx

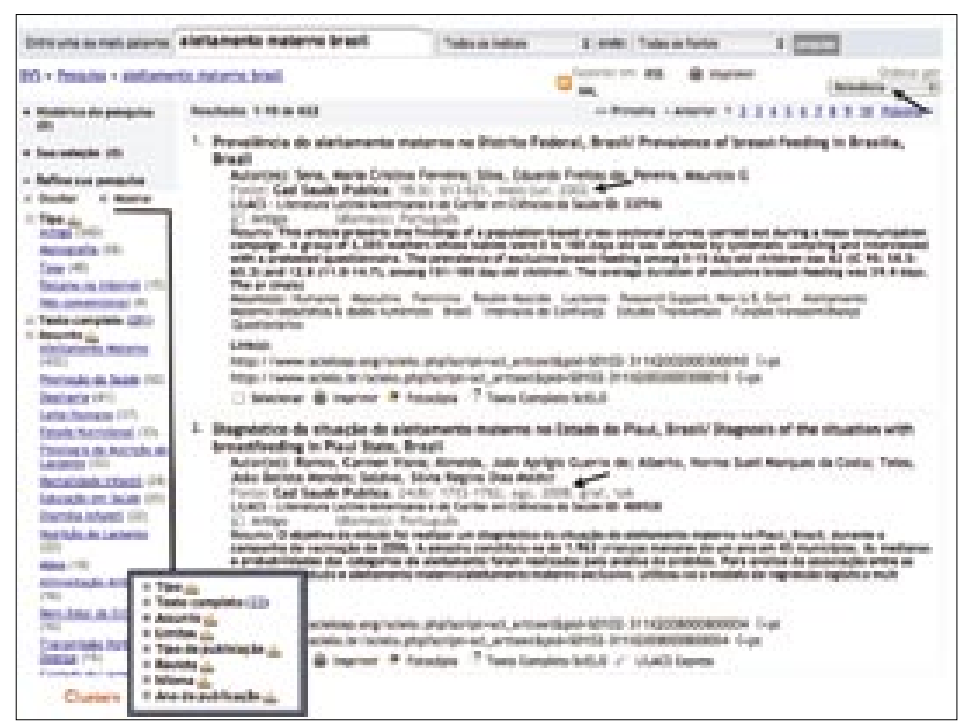


clusters definidos para a coleção da BVS estão mostrados no quadro em destaque. A "clusterização" do resultado funciona basicamente para organização do resultado da busca, mas é, além disso, um recurso excelente para o refinamento da busca. Ao clicar em um dos itens de determinado cluster, por exemplo, o item "promoção da saúde" dentro do cluster "assunto", antecipadamente o usuário já sabe que será apresentada uma lista de 43 documentos relacionados a essa nova busca ("aleitamento materno Brasil" com o assunto "promoção da saúde").

O sistema novamente "clusteriza" e reorganiza o resultado da busca refinada (43 referências de documentos). Esse processo é recursivo, ou seja, é repetido para cada busca.

O sistema de recuperação iAHx já está em uso em alguns portais de serviços de informação do contexto da Biblioteca Virtual em Saúde. Basicamente, as etapas do processo para a implantação do sistema considerando apresentação dos resultados de forma integrada e clusterização são:

- seleção das fontes de informação que serão integradas no sistema de identificação dos principais elementos de dados, principais serviços que podem ser associados, etc.;

- definição da lista de elementos que serão apresentados como clusters no resultado da

\section{FIGURA 4}

Nova "clusterização" do resultado da busca refinada - iAHx

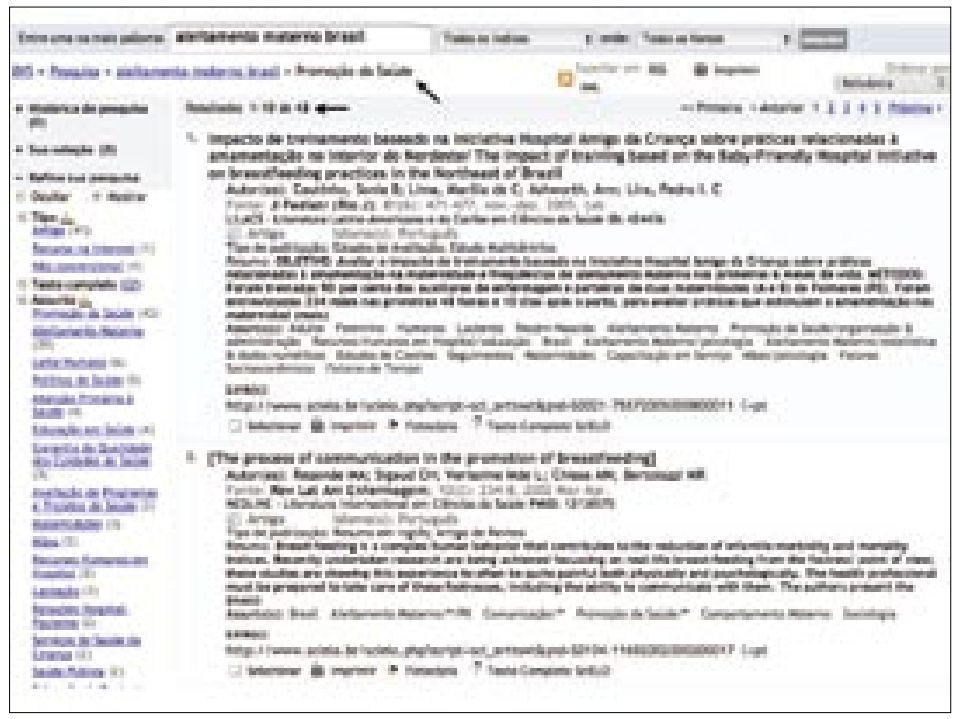

busca. É importante observar que os clusters não são, necessariamente, um espelho dos principais campos de dados das fontes de informação incluídas no sistema de busca. Os clusters podem ser montados em função de um conjunto de dados ou características que definem o cluster. Por exemplo, pode-se apresentar um cluster por nível de evidência dos documentos mesmo que esse dado não esteja registrado na fonte, mas que possa ser gerado através da análise automatizada de um conjunto de elementos presentes na fonte de informação;

- padronização dos valores de elementos comuns entre as fontes de informação, por exemplo, se todas as fontes possuem um elemento que indica qual o idioma do documento, esse valor deve ser padronizado, nesse caso se for utilizada a norma ISO teremos os valores "pt" para português, "es" para espanhol, etc.;

- conversão dos dados das fontes em formato XML seguindo um schema predefinido. O XML resultado dessa conversão deverá conter um subconjunto de metadados que será apresentado no resultado;

- criação de mecanismos que garantam a atualização dessas fontes de informação. Por exemplo, se a fonte de informação está em servidor externo, deve-se criar uma forma, de preferência automática, para a coleta periódica desses dados (harvesting);

- indexação dos metadados: uma vez coletados os metadados em formato XML, será realizada a indexação e "clusterização" dos elementos. O índice resultante desse processo ficará disponível através de webservices em um servidor central;

- disponibilização da interface de consulta: esse módulo é responsável por fazer a comunicação com os webservices disponibilizados no processo anterior e apresentar a informação em formato de páginas web para o usuário realizar as consultas e aplicar os filtros nos valores dos clusters.

\section{CONSIDERAÇ̃̃ES FINAIS}

Considerando o lado dos usuários que buscam informação na $w e b$, espera-se que 
as interfaces de busca sejam eficientes para atraí-los para o uso dos recursos disponíveis, e retê-los para que continuem usando os recursos (Wu; Chung \& Chen, 2008)

De fato, a era digital traz grandes mudanças para os sistemas de recuperação de informação, para os usuários e para os ambientes onde os usuários interagem com os sistemas. Traz também desafios aos usuários para recuperar a informação de forma eficiente e atender às suas necessidades e objetivos (Xie, 2008)

Para o mundo das bibliotecas virtuais e Opacs existem dois grandes desafios ou duas importantes necessidades: disponibilizar os seus conteúdos (fontes de informação) para que estejam visíveis para diferentes interfaces debusca, incluindoos buscadores comoGoogle, e desenvolver sua própria interface de busca para localização da informação dentro da sua coleção (Mi \& Weng, 2008).

Portanto, além disso, conectar os seus usuários com o conteúdo de que eles precisam, quando precisam, não é mais opcional. Os usuários esperam resultados de alta qualidade e que a interface de busca realmente os ajude a encontrar o que estão procurando de maneira rápida e fácil (Wu; Chung \& Chen, 2008).

Os recursos de relevância e de "clusterização" estão presentes nos novos desenvolvimentos de interfaces de busca na web, mas há um grande espaço para aprimoramento e inovações. A aplicação de clusters no processo de busca ainda está muito limitada à estrutura dos campos de dados das fontes de informação. Entretanto, podemos determinar outros clusters de acordo com a área temática da coleção de fontes de informação buscada ou, ainda, permitir que o próprio usuário defina os clusters de seu interesse. Certamente, veremos essas inovações em muito pouco tempo.

Para os portais e sites acadêmicos brasileiros, principalmente as bibliotecas virtuais e Opacs, é importante definir uma nova arquitetura para suas fontes de informação, com integração a partir de recursos de busca e navegação que se aproximem dos recursos utilizados pelos sites e portais buscadores.

\section{BIBLIOGRAFIA}

AGUILLO, I. Internet Invisible: Ios Contenidos Son la Clave. Cindoc-CSIC, 2003. Disponível em: http://internetlab.cindoc.csic.es/cursos/Internet_Invisible2003.pdf.

ESPADAS, J.; CALERO, C.; PIATTINI, M. “Web Site Visibility Evaluation”, in J. Am. Soc. Information Sci. Technol., v. 59, n. 11, set./2008, pp. 1.727-42.

JACSÓ, P."As We May Search: Comparison of Major Features of the Web of Science, Scopus, and Google Scholar Citation-based and Citation-enhaced Databases", in Current Science, v. 89, n. 9, 2005, pp. 1.537-47. Disponível em: http://www.ias.ac.in/currsci/nov102005/contents.htm. .SAVVY Searching - Clustering Search Results. Part I:Web-wide Search Engines", in Online Information Review, v. 31, n. 1, 2007, pp. 85-91.

"SAVVY Searching - Clustering Search Results. Part II: Search Engines for Hihhly Structured Databases", in Online Information Review, v. 31, n. 2, 2007, pp. 234-41.

KOSHMAN, S.; SPINK, A.; JANSEN, B. J. "Web Searching on the Vivisimo Search Engine”, in J. Am. Soc. Information Sci. Technol., v. 57, n. 14, dec./2006, pp. 1.875-87.

MI, J.;WENG, C." Revitalizing the Library OPAC:Interface, Searching, and Display Challenges", in Information Technol. Libraries, v. 27, n. 5, mar./2008, pp. 5-22.

WU, L. L.; CHUNG, Y. L.; CHEN, P.Y." Motivation for Using Search Engines: a Two-factor Model,'in J. Am. Soc. Information Sci. Technol., v. 59, n. 11, set./2008, pp. 1.829-40.

$\mathrm{XIE}, \mathrm{H}$."Shifts in Information-seeking Strategies in Information Retrieval in the Digital Age. A Plannedsituational Model',in Information Research:International Electronic Journal,v.13,n.2,jun./2008,p.22. 